DOI: $10.3901 / J M E .2021 .15 .138$

\title{
基于随机种子的多孔含油保持架建模方法研究
}

\author{
$尹^{\text {婷婷 }^{1}}$ 孙小波 $^{2}$ 间柯 ${ }^{1}$ 洪 $^{1}$ 朱永生 $^{1}$ 李媛媛 $^{2}$ \\ (1. 西安交通大学现代设计与转子轴承系统教育部重点实验室 西安 710049; \\ 2. 洛阳轴承研究所有限公司 洛阳 471039)
}

\begin{abstract}
摘要: 多孔含油保持架凭借其内部微孔隙结构存储和释放润滑介质实现自润滑效应, 对保持架自身微米、亚微米级孔隙结构 的几何建模是其多力场作用过程及自润滑机理分析的前提。针对现有多孔材料建模方法孔隙率大、局部失真等问题，基于冷 压烧结工艺的成型原理, 提出一种面向多孔保持架二维及三维结构的数字化快速建模方法。基于随机种子方法确定颗粒中心 位置及颗粒几何尺寸, 结合堆叠原理模拟颗粒在烧结过程中随机堆叠挤压过程, 采用伪随机数发生器确保多次堆叠过程的颗 粒中心及尺寸的随机性。在此基础之上, 借助 Rhino 软件完成三维建模并与真实多孔含油保持架结构进行对比验证。最后通 过 COMSOL Multiphysics 平台开展多孔保持架二维几何建模及多场耦合分析, 从微孔隙内部的流动与传热过程分析验证了本 文建模方法的可行性与正确性, 为小尺度孔道、小孔隙率三维模型构建提供了新思路。
\end{abstract}

关键词：多孔含油保持架；随机种子理论；建模方法；模型验证

中图分类号: TG156

\section{Research on Modeling Method of Porous Oil-containing Cage Based on Random Seeds}

\author{
YIN Tingting $^{1} \quad$ SUN Xiaobo $^{2} \quad$ YAN Ke $^{1}$ HONG Jun $^{1} \quad$ ZHU Yongsheng $^{1}$ LI Yuanyuan ${ }^{2}$ \\ (1. Key Laboratory of Education Ministry for Modern Design and Rotor-Bearing System, Xi'an Jiaotong \\ University, Xi'an 710049; \\ 2. Luoyang Bearing Research Institute Co. Ltd, Luoyang 471039)
}

\begin{abstract}
The self-lubricating effect of porous oil-containing cage is realized by storing and releasing the lubricating medium in the micro-pore structure. The geometric modelling of the micron and sub-micron pore structure of the cage is the premise of researching its multi-force-field action process and analysing its self-lubrication mechanism. Aiming at the problems of the current porous material modelling methods, such as large porosity and local distortion, a rapid digital modelling method for two-dimensional and three-dimensional structures of porous cages is proposed, based on the forming principle of the powder metallurgy process. According to the random seed method, the centre position and geometric size of particles are determined, and the sintering process of random stacking and squeezing of particles is simulated by using the stacking principle. In addition, a pseudo-random number generator is used to ensure the randomness of the multiple stacking processes of the particle centre position and size. Build on this, three-dimensional modelling, which is completed through the Rhino software, is compared and verified with the actual structure of the porous oil-containing cage. Finally, two-dimensional geometric modelling and multi-field coupling analysis of the porous cage are carried out by the COMSOL Multiphysics platform. The feasibility and correctness of the modelling method are verified via analysing the flow and heat transfer process inside the micropores, which provides new ideas for the analysis of three-dimensional structures of small-scale channels and small porosities.
\end{abstract}

Key words: porous oil-containing cage; random seed theory; modeling method; model validation

*国家自然科学基金优秀青年科学基金(52022077)资助项目。20200803 收到 初稿, 20201119 收到修改稿 


\section{0 前言}

多孔保持架因其优异的自润滑特性使其在航 空航天、精密回转等重大装备支撑轴承中广泛应 用。在轴承服役过程中, 存储在多孔保持架微米 级、亚微米级孔隙中的润滑介质依靠轴承自身运 转产生的离心力、热效应和孔隙的毛细力实现润 滑油的溢出-吸入自循环式供给并实现轴承润 滑 $^{[1]}$ 。可以看出, 润滑介质在微小孔隙内部的流动 特性、润滑介质与孔隙的作用力以及润滑介质与 多孔材料间的热流耦合效应是影响多孔保持架维 持良好自润滑性能的关键, 特别是在真空、极温 环境下, 润滑介质与微孔隙的相互耦合作用进一 步突显, 成为制约多孔含油保持架润滑性能以及 轴承服役性能的关键。

目前国内外的含油轴承领域无针对多孔含油保 持架微观参数的设计标准, 仅有为满足轴承安装设 计的宏观尺寸标准。因此多孔含油保持架的多孔参 数对含油轴承润滑性能之间的关系不明确, 需要进 一步研究。针对多孔含油保持架润滑的研究文献主 要集中在试验研究, 如间普选等 ${ }^{[2-4]}$ 分别试验研究了 多孔保持架不同孔隙率、材料基体等对其宏观摩擦 学与润滑性能的影响。由于缺乏有效的几何建模手 段, 导致难以从机理上分析多孔保持架微孔隙内润 滑介质自润滑过程。为了解决这一问题, 魏博熙 ${ }^{[5]}$ 、 $\mathrm{WANG}^{[6]}$ 使用 X-ray 技术对多孔材料进行了 $\mathrm{CT}$ 扫描 重构，获得了简化的多孔模型。基于 X-ray 扫描重 构数据, 文献[7-8]提取多孔模型的几何形状和互连 性数据, 利用最大球法将孔道近似等效为孔和喉, 建立了多孔材料几何模型。由于多孔含油保持架一 般由冷压烧结成型, 其内部孔径在 $0.5 \sim 5.0 \mu \mathrm{m}^{[9]}$, 而目前 X-ray 技术的最大分辨率为 $0.699 \mu \mathrm{m}$, 导致 大量的微孔隙结构难以扫描识别, 重构模型严重 失真。

为了获得可用于内部流动分析的三维数字化 孔隙模型, LI 等 ${ }^{[10]}$ 将多孔结构简化为具有简单立 方堆积的 $3 \mathrm{D}$ 晶格结构, 设计一个中心孔与在横向 和垂直方向上拉伸的六个喉部相互连接。此方法 虽然保留了多孔结构的孔与喉特征, 但缺失了随 机孔的分布形态, 与真实多孔结构在孔径、孔分
布方面均有较大差异。对此, 文献[11]采用随机生 长四参数法, 生成多孔介质二维图形, 并结合多 孔材料的扫描电镜(SEM)图对生成的轮廓图进行 处理, 然而所建模型孔道之间均相互连通并均匀 分布, 与实际多孔结构的复杂随机性仍有较大差 异。文献[12]采用球形颗粒来模拟多孔材料, 在立 方体单元中随机排布分散颗粒，生成了具有随机 分布与随机大小多孔模型。但此模型孔隙率较大 $(>30 \%)$, 不适用于多孔含油保持架等微米、亚微 米级多孔结构的精确建模。

通过对多孔保持架冷压烧结成型过程分析可 以看出, 多孔保持架的多孔结构是由原料粉末颗粒 经机械压实后在一定温度下进行烧结, 聚合物颗粒 融化粘接后，颗粒之间的缝隙形成的微观通道。对 此, 本文提出了一种基于随机种子理论的多孔保持 架建模方法, 通过在限定空间中随机排布规定尺寸 或者随机尺寸的种子, 并基于颗粒堆叠与布尔运算 获得具有随机特点的模型, 在 Rhino 框架以及 COMSOL Multiphysics 平台分别开展三维、二维建 模, 获得了具有高还原度的多孔介质模型。在此基 础上, 利用 COMSOL Multiphysics 平台模拟了润滑 介质在多孔保持架微孔隙内部离心力、热膨胀耦合 作用下的流动状态，验证了本文建模方法的可行 性, 为多孔含油保持架润滑性能机理研究提供了新 思路。

\section{1 随机种子的模型理论}

\section{1 随机数发生器原理}

随机数是指一个随机变量 $X$ 的抽样序列, 如 式(1), 其最重要的特性是随机产生数与序列中已有 的任意一个数都毫无关系，如 $x_{n}$ 与序列 $x=x_{1}, x_{2}, \cdots, x_{n-1}$ 均无任何关系。

$$
x=x_{1}, x_{2}, \cdots, x_{n}
$$

一般使用数学公式递推产生各种分布的随机 数。例如使用 “乘同余法”, 可以产生在区间 $(0$, $M)$ 上的整型均匀随机数如式 $(2)^{[13]}$ 。

$$
x_{K}=\lambda x_{K-1}(\bmod M), K=1,2,3, \cdots
$$

式(2)称为同余式, $\lambda$ 是乘子, $x_{0}$ 是初始值, $M$ 
称为模; $\bmod M$ 是取模运算, 其方程如式(3)。

$$
x_{K}=\left\{\begin{array}{l}
\lambda x_{K-1} \quad \lambda x_{K-1}<M \\
\lambda x_{K-1}-n M \quad \lambda x_{K-1} \geqslant M
\end{array}\right.
$$

式中, $n$ 为整数, 经取模运算可使 $x_{K}$ 的值落在区间 $(0, M)$ 上。因此只要给定 $M, \lambda, x_{0}$, 就可以生成一 组落在 $(0, M)$ 上的随机数。此类随机数实际上是通 过一个固定的、可以重复的计算方法即伪随机数发 生器 $(\operatorname{rand}())$ 产生, 它们具有类似于随机数的统计特 征。本文随机种子的产生实际上就是利用了伪随机 数发生器原理。

\section{2 随机种子的模型理论}

多孔含油保持架材料的孔隙大小及分布取决于 原料配比与制备工艺, 因在烧结压制过程中, 原料 粉末受压制压力、烧结温度、保温时间等的影响发 生变形、粘接, 最后由颗粒之间的孔隙形成多孔结 构。基于多孔保持架的制备工艺过程, 结合伪随机 数发生器原理, 提出一种建立多孔介质三维实体模 型与多孔介质二维截面模型的随机种子建模方法, 具体如下。

(1) 根据多孔保持架的原料粒径 $\left(d_{g}\right)$ 所占比例 $(P \%)$, 分配种子的直径大小 $\left(d_{s}\right)$ 以及数量 $\left(N_{s}\right)$, 即满 足 $d_{s} \approx d_{g}, N_{s 1}: N_{s 2}: \cdots=p_{1} \%: p_{2} \%: \cdots$ 。

(2) 设定模型规格即建模空间大小 (边长设置为 $L$ ), 作为播散种子的空间 (三维为 $L^{3}$, 二维为 $L^{2}$ ) 以 及限定种子的投放位置 $\left(x_{m}, y_{m}\right)$ 满足式(4)

$$
\left(x_{m}, y_{m}\right) \in L^{3}\left(L^{2}\right)
$$

(3) 利用伪随机数发生器 $(\operatorname{rand}())$ 产生种子的随 机位置坐标 $\left\{\left(x_{1}, y_{1}\right),\left(x_{2}, y_{2}\right), \cdots,\left(x_{m}, y_{m}\right)\right\}$, 并在位置 点上生成规定尺寸 $\left(d_{s}\right)$ 或者随机尺寸 $\left(d_{\mathrm{rand}}\right)$ 的球形 种子。

(4) 检查球形种子是否重叠, 如式(5), 如果重 叠则进行布尔运算的并集操作 $\left(A_{\text {seed }} \cup B_{\text {seed }}\right)$, 将两 个实体合并为一个实体

$$
\left\{\begin{array}{l}
\left|x_{m}-x_{m-1}\right|<\min \left(0.5 d_{s m}, 0.5 d_{s(m-1)}\right) \\
\left|y_{m}-y_{m-1}\right|<\min \left(0.5 d_{s m}, 0.5 d_{s(m-1)}\right)
\end{array}\right.
$$

(5) 检查球形种子是否有溢出建模空间, 如 式(6), 如果溢出则进行布尔运算删除溢出的部分 $\left(A_{\text {seed }}-C_{U}\left(L^{3}\right)\right)$ 或者 $\left(A_{\text {seed }}-C_{U}\left(L^{2}\right)\right)$

$$
\begin{cases}x_{m}+0.5 d_{s m}>L & x_{m}-0.5 d_{s m}<0 \\ y_{m}+0.5 d_{s m}>L & y_{m}-0.5 d_{s m}<0\end{cases}
$$

重复进行步骤(4)与步骤(5), 直到所有重叠球形 种子合并为一个实体、溢出的球形种子均被切断删 除, 最后利用建模空间 (三维为 $L^{3}$, 二维为 $L^{2}$ ) 减去 种子堆积模型, 获取种子间的空隙, 生成最终的多 孔介质模型。

\section{2 多孔介质三维实体建模}

\section{1 建模过程}

基于随机种子的模型理论, 利用 Rhino 设计工 具开展了多孔材料三维实体建模研究, 利用软件的 参数化建模插件(Grasshopper)和曲面建模(Nerbs)生 成多孔结构的实体模型的建模流程图如图 1。首先 以特定空间边长 $L$ 的立方体定义建模空间 $L^{3}$, 如 图 1 中(1)部分的 Center Box 模块。

在规定的建模空间 $L^{3}$ 内, 利用参数化与可视化 的模块 Populate 3D 随机填充种子如图 1 中的(2)部 分。其中区域(Region)参数连接 Center Box 的 Box 选项以约束添加随机点的空间, 数量(Count)参数根 据多孔保持架的原料配比设置为 $N_{s}$, 种子数列(Seed) 参数基于随机数发生器原理选定一类种子的随机排 列方式 $\operatorname{rand}\left\{\left(x_{1}, y_{1}\right),\left(x_{2}, y_{2}\right), \cdots,\left(x_{m}, y_{m}\right)\right\}$ 。此选定原 理是针对相同数值的 seed 为同种随机分布, 改变 seed 数值则相当于重新洗牌产生另一种 $\operatorname{rand}\left\{\left(x_{11}, y_{11}\right),\left(x_{22}, y_{22}\right), \cdots,\left(x_{m m}, y_{m m}\right)\right\}$ 。如此反复选 定不同的随机排布方式，模拟微小空间内不同粉末 的随机堆叠如图 2 所示。

Sphere 模块是基于基础(Base)参数选项确定的 基点位置 rand $\left\{\left(x_{1}, y_{1}\right),\left(x_{2}, y_{2}\right), \cdots,\left(x_{m}, y_{m}\right)\right\}$ 和直径 参数 $d_{s m}$ 建立圆球实体模型, 最后把所需模拟的种子 直径 $d_{s m}$ 分组搭建即多组 population3D 模块与 sphere 模块并联，生成初始模型。因部分在立方体边缘的 随机点生成的球体溢出边界且各球体还处于相互独 立的状态即满足式(5)、(6), 则反复运用布尔运算的 并集 $\left(A_{\text {seed }} \cup B_{\text {seed }}\right)$ 、差集运算 $\left(A_{\text {seed }}-C_{U}\left(L^{3}\right)\right)$ 如图 1 中的(3)部分, 删除多余的实体, 合并重叠的实体, 使其变成一个整体实体模型后, 利用建模空间 $L^{3}$ 减 去种子堆积模型, 获取空隙模型, 得到完整的多孔 介质三维实体模型如图 3 所示。 


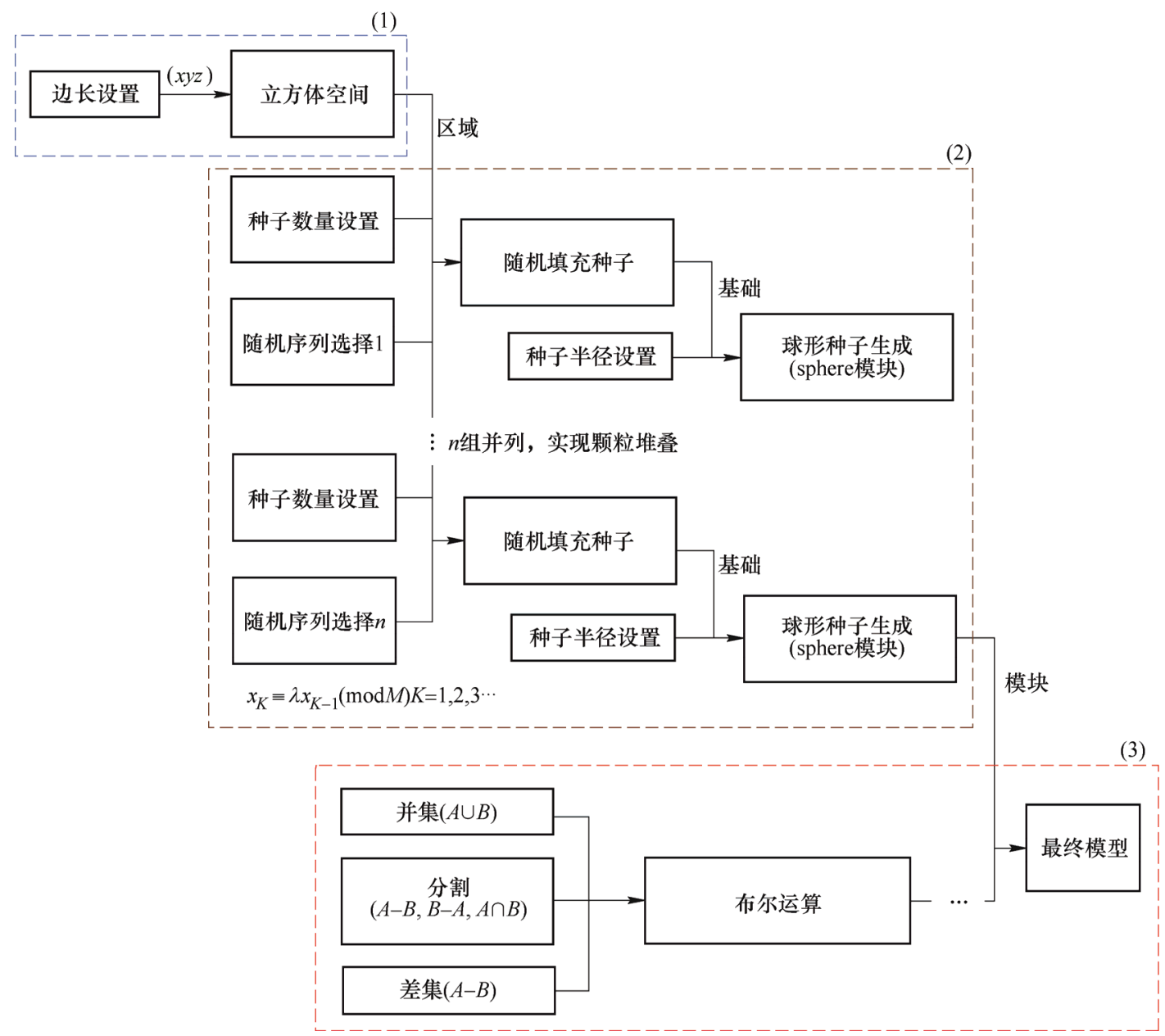

图 1 Rhino 建模流程图

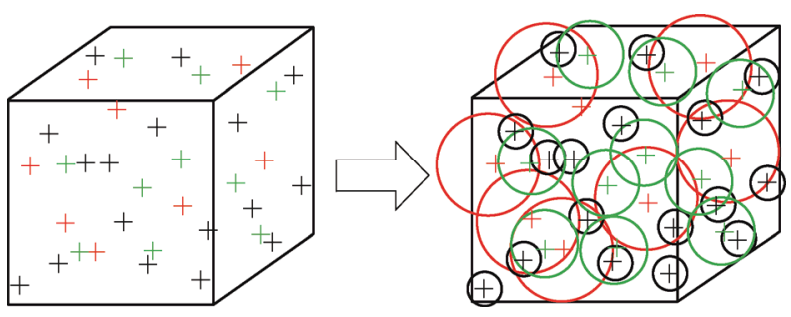

图 2 随机点处生成圆球示意图

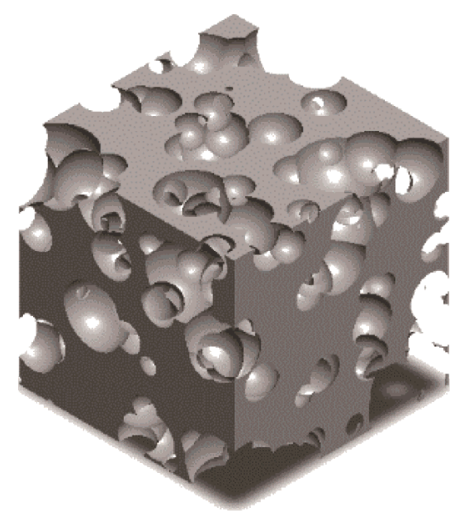

图 3 多孔材料局部三维实体模型

\section{2 孔隙特征分析}

基于建立的多孔介质三维实体模型，利用 $3 \mathrm{D}$ 打印软件计算多孔介质三维实体模型的质量，同时 计算同材料等体积立方体的总质量, 最后计算得到 多孔介质结构的孔隙率如式(7)。

$$
\gamma=\frac{m_{\text {pore }}}{m_{\text {total }}} \times 100 \%
$$

由此初步计算建立的多孔介质三维实体模型 的孔隙率为 $25 \%$, 而采用压录仪对常用多孔含油 保持架进行测量, 得到孔隙率为 $21 \%$, 二者相差 $4 \%$, 在允许的误差范围内。同时, 采用日本 $\mathrm{S}-3000 \mathrm{~N}$ 型扫描电子显微镜(SEM)对多孔含油保 持架材料进行扫描, 得到多孔含油保持架材料 的 SEM 图像如图 4 所示, 其中图中标尺分别为 $10 \mu \mathrm{m} 、 1 \mu \mathrm{m}$ 。

为了能清晰地表现出多孔三维实体模型中的孔 隙分布, 从图 3 中选取颗粒堆积模型的一个截面如 图 5 所示进行观察。 


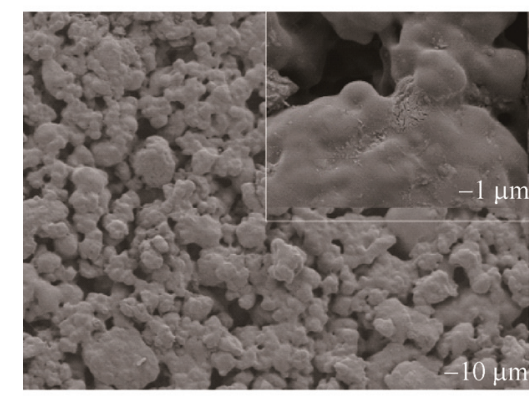

图 4 多孔保持架材料的 SEM 图像

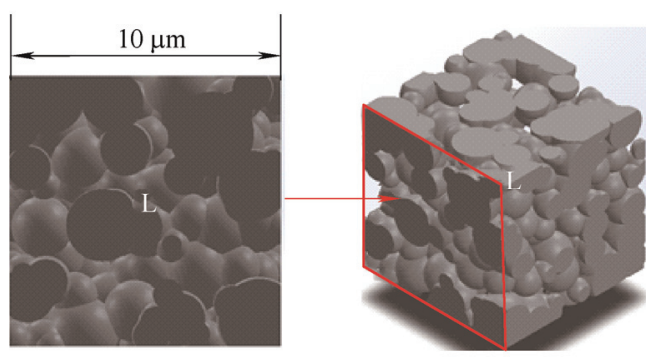

图 5 颗粒堆积模型的截面提取图

将图 5 与图 4 进行对比观察可知, 建立的多孔 局部三维实体模型与多孔保持架的 SEM 图像呈现 的多孔形态相似, 颗粒与颗粒之间相互重叠, 颗粒 未重叠的空隙即为多孔结构, 验证了模型结构的合 理性, 为多孔含油保持架润滑性能的模拟仿真研究 提供新方向。

\section{3 多孔介质二维截面建模}

利用有限元法对多孔介质三维实体模型进行 多孔渗流分析时, 由于存在很多极端狭窄的区域, 极易出现面或域小于最小网格单元的情况, 导致 模型的网格单元数量巨大且网格质量低, 计算结 果极易出现发散错误。此外, 针对三维模型仿真 计算自由度是二维模型计算的 5 倍, 计算量大, 难以都到准确的仿真结果。因此, 为了便于开展 多孔模型的建模与仿真分析, 在建立多孔介质三 维实体模型的基础上, 利用随机种子理论在 COMSOL Multiphysics 软件内建立不同孔隙率的 多孔介质二维模型。

\section{1 建模过程}

基于随机种子的模型理论, 利用 COMSOL Multiphysics 模型开发器开展了多孔材料的二维截 面建模研究, 以调用包含 Math.random 类在内的标 准 $\mathrm{Java}^{\circledR}$ 类的方式完成多孔材料的复杂二维截面 模型的建模，算法原理流程如图 6 所示。

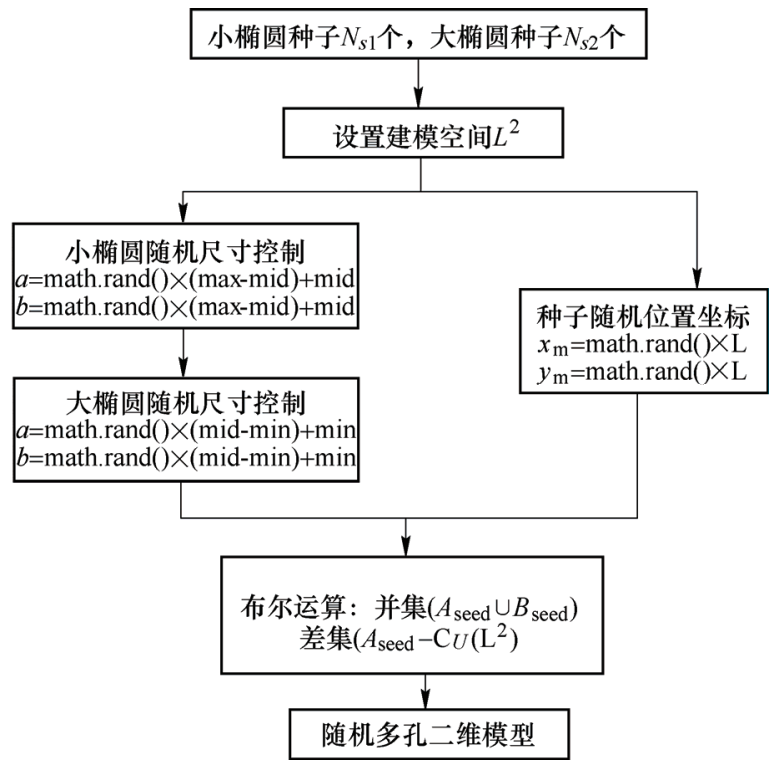

图 6 算法流程图

首先设置种子数量 $N_{s}$, 以及规定建模空间 $L^{2}$ (本文 $L=100 \mu \mathrm{m}$ )。文献[14]利用 X-ray CT 技术对多 孔保持架三维内部结构进行了扫描观测, 得出多孔 保持架内的小孔占绝大部分, 小孔密集排布是孔连 通的主要原因。因此, 在参考原料粒径 $d_{g}$ 的基础上, 将种子尺寸分为两类, 即小尺寸种子 $N_{s 1}$ 个与大尺 寸种子 $N_{s 2}$ 个, 且满足 $N_{s 1}>N_{s 2}$ 。此外, 多孔保持 架在压制成型过程中, 原料颗粒受挤压变形, 单从 截面上看，颗粒形状已不是圆形。

为了提高多孔介质二维截面模型的还原度, 将 球形种子转为椭圆种子, 并通过 $d_{s} \times d_{s} \approx a_{s} \times b_{s}$ 保证 建模原理一致。本文设置种子形状为椭圆, 因伪随 机数发生器的 $\operatorname{random}()$ 函数的返回值是大于等于 0.0 且小于 1.0 之间的随机双精度浮点数, 生成的 种子尺寸 $a_{s}, b_{s} \in\left[d_{s \text { min }}, d_{s \text { max }}\right]$ 。另一方面, 利用伪随 机数发生器产生在建模空间 $L^{2}$ 内种子的随机中心 坐标 $\operatorname{rand}\left\{\left(x_{1}, y_{1}\right),\left(x_{2}, y_{2}\right), \cdots,\left(x_{m}, y_{m}\right)\right\}$ 。随机尺寸的 椭圆种子在建模空间内随机堆叠过程与原料颗粒在 保持架模具内压制成型过程一致，还原了多孔材料 内多孔结构的真实形成过程。

根据随机种子建模理论的式(5)、(6), 检查随机 种子在建模空间内的分布情况, 利用布尔运算对重 叠的图形求并集 $\left(A_{\mathrm{seed}} \cup B_{\mathrm{seed}}\right)$ 以及对溢出的图像求 差集 $\left(A_{\text {seed }}-C_{U}\left(L^{3}\right)\right)$, 最后以建模空间 $L^{2}$ 减去种子 堆积模型生成孔隙轮廓图。因孤立孔内无润滑油的 流动, 所以删除孤立孔以减少后期模拟仿真的网格 计算量。本文建立了与多孔材料 SEM 图高度相 像的四个孔隙率(包括 $0.1534 、 0.1913 、 0.249$ 1、 $0.2848)$ 的多孔介质二维截面模型, 如图 7 所示。 


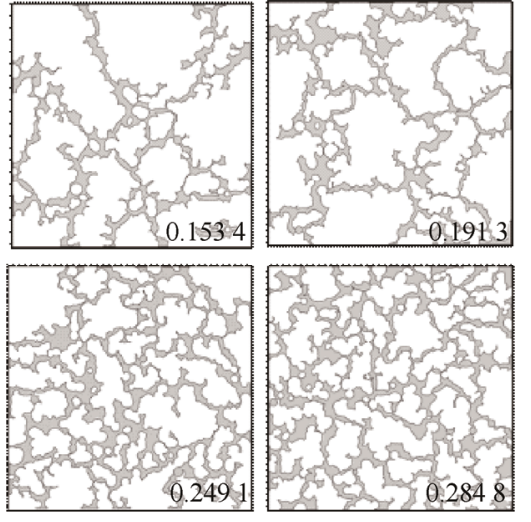

图 7 多孔介质二维截面模型

\section{2 孔隙特征分析}

对多孔保持架材料的 SEM 图像进行图像分割 得到孔隙率为 $13.39 \%$ 的多孔结构二值图如图 8 所 示, 白色部分为孔隙, 黑色部分为多孔保持架固体 材料。

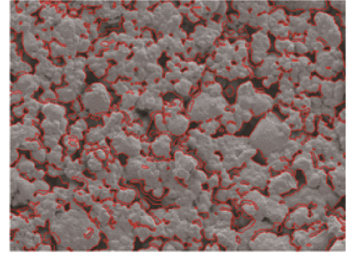

(a) 图像分割

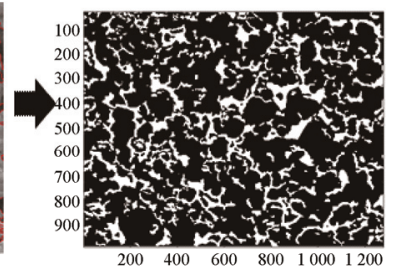

(b) 二值图
图 8 孔隙结构二值图

图 9 为建立的孔隙率为 $15.34 \%$ 的未经修改的多 孔介质二维截面模型原图, 灰色部分为孔隙, 白色 部分为多孔含油保持架固体材料。将图 8 与图 9 进 行对比可知, 建立的多孔二维模型基本上承袭了多 孔保持架材料的原型, 在定量指标 (孔隙率、孔尺寸) 与定性指标(孔形态、孔分布)方面均具有非常高的 还原度。

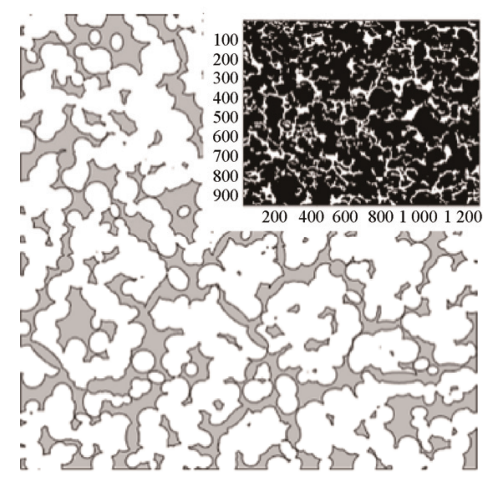

图 9 多孔介质二维模型原图

\section{4 模型仿真分析与验证}

以 7008C 轴承的多孔含油保持架(聚酰亚胺材 料)为背景，建立的多孔介质二维模型为基体，依托 COMSOL Multiphysics 多物理场耦合软件, 研究多 孔含油保持架在离心力以及热效应的影响下，润滑 油的流动状态变化规律, 参数设置如表 1 , 其中环 境温度为 $20{ }^{\circ} \mathrm{C}$, 转速为 $2000 \mathrm{r} / \mathrm{min}$, 润滑油型号 为 4123 。

\section{表 1 仿真参数设置}

\begin{tabular}{ccc}
\hline \multirow{2}{*}{ 参数 } & \multicolumn{2}{c}{ 数值 } \\
\cline { 2 - 3 } & 动力黏度 $/(\mathrm{Pa} \cdot \mathrm{s})$ & 边界温升 $/{ }^{\circ} \mathrm{C}$ \\
\hline 离心力 & 0.79 & 0 \\
离心力与热 & 0.54 & 5 \\
\hline
\end{tabular}

\section{1 离心力仿真}

\subsection{1 方程及边界条件}

因多孔含油保持架内的孔径属于微米级、亚微 米级, 润滑油在孔道内流动非常缓慢, 属于雷诺数 比较低的流体流动, 因此选择“蠕动流”作为润滑油 受离心力影响向外流动的物理场接口。

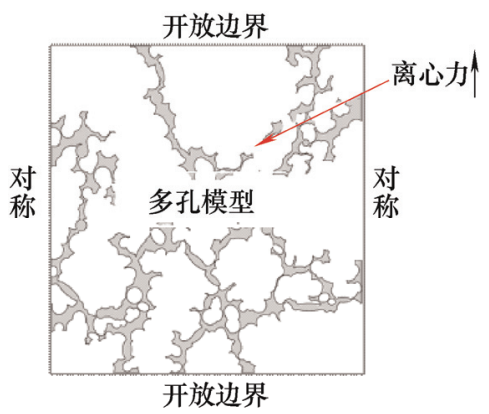

图 10 离心力仿真边界条件设置

计算方程为包括动量守恒的斯托克斯方程 (Navier-Stokes)和用于质量守恒的连续方程, 忽略 Navier-Stokes 方程中的惯性项。因多孔介质内压力 变化大，设置流体为弱可压缩流动。设置边界条件 如图 10, 并在 $y$ 向添加离心力如式(8), 其中 $\omega=\nu \times \pi / 30$

$$
F_{c}=\rho(y+0.028[m]) \omega^{2}\left[N / m^{2}\right]
$$

式中, $\rho$ 为润滑油的密度, 由材料属性确定; 保持 架的半径为 $0.028 \mathrm{~m}$, 由 $7008 \mathrm{C}$ 轴承尺寸确定; $v$ 为 转速。

\subsection{2 仿真结果分析}

选择瞬态求解器以研究流体流动中的非定常流 
场以及压力场随时间变化的情况, 设置时间步长为 $0.025 \mathrm{~s}$ 、计算时间 $5 \mathrm{~s}$, 模拟多孔模型在离心力作用 下润滑油的流动状态, 包括流速及压力分布, 得到 $5 \mathrm{~s}$ 时的流动状态结果如图 11 所示, 依次为 0.153 4、 $0.1913 、 0.2491 、 0.2848$ 孔隙率的多孔模型的速度 以及压力分布。
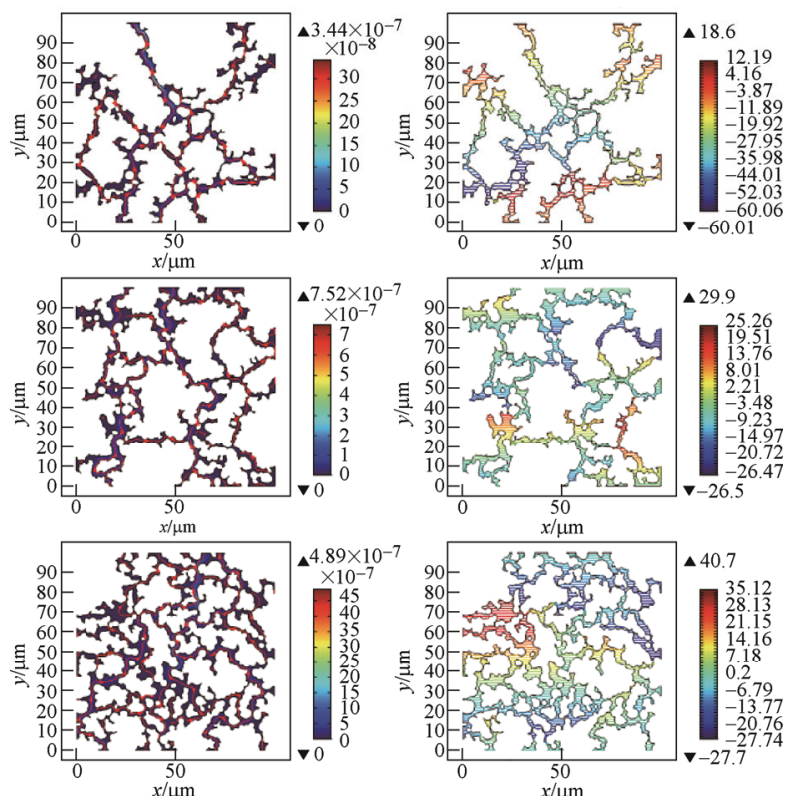

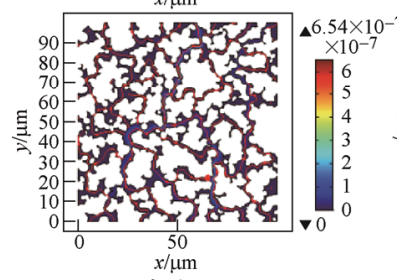

(a) 流速 $(\mathrm{m} / \mathrm{s})$

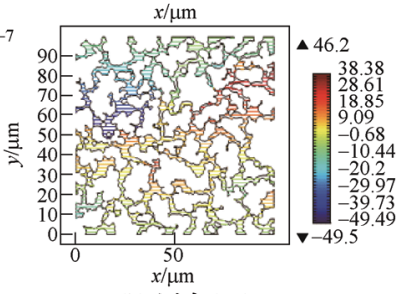

(b) 压力 $(\mathrm{Pa})$
图 11 多孔模型流速以及压力分布

从图中关于多孔介质微孔隙内部流动速度和压 降变化可以看出, 多孔介质内部流场分布总体符合 多孔渗流规律, 即压差较大的地方流速增大。此外, 微孔隙分布与几何尺寸的差异导致局部的流动速度 和压降显著变化, 宏观的摩擦学试验以及孔隙率参 数难以准确反映多孔介质内部的流动机理, 而本文 的建模方法为多孔保持架的润滑机理研究提供了有 力的分析工具。

\section{2 离心力与热效应耦合分析}

\subsection{1 方程及边界条件}

多孔含油保持架内润滑油流动同时受到离心力 以及摩擦温升影响, 有必要将离心力与温度变化耦 合起来计算。因此在 “蠕动流” 接口的基础上, 增 添 “流体传热” 接口用于模拟润滑油的流动传热, 方程为广义的传热方程即能量守恒方程。

多物理场耦合包括流动耦合和温度耦合, 其中 流动耦合将流动方程中的 $u$ 和 $p$ 应用到传热方程中,
温度耦合将 $T$ 应用到流动方程中即考虑温度对材料 属性(流体黏度、密度等)的影响。以此双向耦合的方 式模拟多孔含油保持架在离心力与热效应的综合影 响下内部润滑油的流动状态, 边界条件设置如图 12, 在入口边界设置相对于环境温度高 $5{ }^{\circ} \mathrm{C}$ 的上游流体 温度以表示多孔含油保持架因摩擦生热的温升。

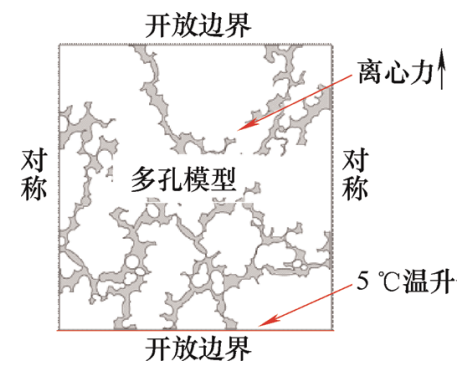

图 12 离心力与摩擦生热耦合仿真边界条件设置

\subsection{2 仿真结果分析}

选择瞬态求解器以研究流体流动中的非定常流 场、压力场以及温度场随时间变化的情况。因流体 中发生对流换热, 模型温度达到稳定的时间较长, 因此设置时间步长为 $0.025 \mathrm{~min}$, 计算时间为 $3 \mathrm{~min}$ 。 模型计算得到的流速以及压力分布与只考虑离心力 作用的仿真结果类似, 这里不再赘述。对比两次仿 真模型的出口边界流量, 得到结果如表 2 。

表 2 多孔介质模型的边界流量 $\left(\mathrm{m}^{2} / \mathrm{s}\right)$

\begin{tabular}{ccc}
\hline 孔隙率 & $2000 \mathrm{r} / \mathrm{min}$ & $2000 \mathrm{r} / \mathrm{min} \& \Delta T=5{ }^{\circ} \mathrm{C}$ \\
\hline 0.1534 & $1.59 \times 10^{-13}$ & $2.11 \times 10^{-10}$ \\
0.1913 & $4.00 \times 10^{-13}$ & $3.92 \times 10^{-10}$ \\
0.2491 & $2.68 \times 10^{-13}$ & $3.63 \times 10^{-10}$ \\
0.2848 & $4.50 \times 10^{-13}$ & $6.01 \times 10^{-10}$ \\
\hline
\end{tabular}

当只考虑离心力作用即只添加边界条件为转速 $2000 \mathrm{r} / \mathrm{min}$ 时, 出口边界流量大小在 $10^{-13}$ 的数量级, 而在此基础上, 考虑摩擦生热的影响即添加边界条 件为 $5{ }^{\circ} \mathrm{C}$ 温升后出口边界流量大小变为 $10^{-10}$ 的数 量级, 远远超过离心力的作用效果。因此, 相比于 离心力作用, 多孔含油保持架内的润滑油受热溢出 表面的效果更显著, 与文献[15]中多孔保持架宏观 摩擦学性能的试验现象相吻合, 进一步说明基于随 机种子理论的多孔介质建模方法的可行性。

\section{5 结论}

（1）依据多孔保持架制备工艺原理，提出了一 种基于随机种子理论的微米、亚微米级多孔介质的 数字化建模方法。

（2）基于上述建模方法, 利用 Rhino 工具建立 了多孔介质的三维实体模型, 并与保持架 SEM 图像 作对比验证了模型的合理性。 
（3）基于上述建模方法, 利用 COMSOL Multiphysics 工具建立了多孔介质不同孔隙率的二 维模型, 并与保持架二值图对比, 表明了本文建模 方法在定量指标(孔隙率、孔尺寸)与定性指标(孔形 态、孔分布)方面的高可靠性。

(4) 基于所建立的二维模型, 开展了多孔介质 内部多物理场耦合分析, 分析了离心力以及热效应 对多孔介质内部流动特性的影响, 与试验结果对比 进一步验证了本文建模方法的可行性与准确性。

\section{参 考 文 献}

[1] ZHANG D, WANG T M, WANG Q H, et al. Selectively enhanced oil retention of porous polyimide bearing materials by direct chemical modification[J]. Journal of Applied Polymer Science, 2017, 134(29): 1-7.

[2] 问普选, 朱鹏, 谷和平, 等. 热塑性聚酰亚胺多孔材料 的制备工艺的探讨 [J]. 润滑与密封, 2007, 32(1): 79-82. YAN Puxuan, ZHU Peng, GU Heping, et al. Study of preparation technology for thermoplastic polyamide porous material[J]. Lubrication Engineering, 2007, 32(1): 79-82.

[3] 邱优香, 王齐华, 王超, 等. 多孔聚酰亚胺含油材料的 储油性能及摩擦学行为研究 [J]. 摩擦学学报, 2012, 32(6): 538-543.

QIU Youxiang, WANG Qihua, WANG Chao, et al. Oil-containing and tribological properties of porous polyimide containing lubricant oil[J]. Tribology, 2012, 32(6): 538-543.

[4] 石晓婷. 多孔 PI/PTFE 复合材料摩擦学性能研究[D]. 秦皇岛: 燕山大学, 2016 .

SHI Xiaoting. Study on the tribological propertites of porous PI/PTFE composites[D]. Qinhuangdao: Yanshan University, 2016.

[5] 魏博熙. 应用数字岩心技术模拟高温高压气水渗流[D]. 成都: 西南石油大学, 2017.

WEI Boxi. Using digital core technology to simulate gas-water relative permeability under high temperature and high pressure[D]. Chengdu: Southwest Petroleum University, 2017.

[6] WANG J, ZHANG L, ZHAO J, et al. Variations in permeability along with interfacial tension in hydrate-bearing porous media[J]. Journal of Natural Gas Science\& Engineering, 2018, 51: 141-146.

[7] DONG H, BLUNT M. Micro-CT imaging and pore network extraction[D]. London : Earth Science and
Engineering of Imperial College, 2008.

[8] DONG H, BLUNT M. Pore-network extraction from micro-computerized-tomography images[J]. Physical Review E, 2009, 80(3): 1-11.

[9] 张迪, 王超, 卿涛, 等. 空间用多孔聚合物轴承保持架 材料研究进展 [J]. 机械工程学报, 2018, 54(9): 17-26. ZHANG Di, WANG Chao, QING Tao, et al. Research progress of porous polymer bearing retainer materials used in aerospace[J]. Journal of Mechanical Engineering, 2017, 45(5): 473-476.

[10] LI G Y, DAI S, ZHAN L T, et al. A pore-scale numerical investigation of the effect of pore characteristics on flow properties in soils[J]. Journal of Zhejiang University, 2019, 20(12): 961-978.

[11] 岳亚美. 空间微量润滑条件下润滑介质的渗流和导流 行为研究 $[D]$. 哈尔滨: 哈尔滨工业大学, 2018 .

YUE Yamei. Research on percolation and conduction behavior of lubrication medium on under minimum quantity lubrication in space[D]. Harbin: Harbin Institute of Technology, 2018.

[12] 郑建军, 张放, 周欣竹, 等. 多孔材料水渗透系数预测 的随机行走法 [J]. 浙江工业大学学报, 2017, 45(5): 473-476.

ZHENG Jianjun, ZHANG Kang, ZHOU Xinzhu, et al. A random walk method for predicting the water permeability coefficient of porous materials[J]. Journal of Zhejiang University of Technology, 2017, 45(5): 473-476.

[13] 吕志. 第五讲随机数的产生 $[\mathrm{J}]$. 数理统计与管理, 1985(3): 37-45.

LÜ Zhi. Lesson 5 Generation of random numbers[J]. Mathematical Statistics and Management, 1985(3): 37-45.

[14] YIN T T, LI Y Y, YAN K, et al. Effect of pore parameters on lubrication performance of oil-containing cage[J]. Mechanisms and Machine Science, 2019, 77: 1124-1135.

[15] 赵华俊. 采用多孔聚酰亚胺提高滚动轴承贫油润滑性 能的研究[D]. 南京: 南京航空航天大学, 2017.

ZHAO Huajun. Study on improving the performance of rolling bearing at starved lubrication by porous PI[D]. Nanjing : Nanjing University of Aeronautics and Astronautics, 2017.

作者简介: 尹婷婷, 女, 1995 年出生。主要研究方向为多孔含油保持架 的润滑机理研究。

E-mail: ytt0225@stu.xjtu.edu.cn

闵柯(通信作者), 男, 1984 年出生, 博士, 教授, 博士研究生导师。主 要研究方向为轴承润滑与热特性分析。

E-mail: yanke@mail.xjtu.edu.cn 